\title{
Optimal Settings for Frequency-Selective Measurements Used for the Exposure Assessment Around UMTS Base Stations
}

\author{
Christof Olivier and Luc Martens, Member, IEEE
}

\begin{abstract}
To estimate the exposure around a base station, the frequency-selective electromagnetic field measurement with a spectrum analyzer and antenna is the most appropriate method. In this paper, a theoretical model for the wideband code division multiaccess signal, which is measured by a spectrum analyzer, is extended to the measurement of the signal used in the universal mobile telecommunications system (UMTS), where the transmitted signal is subject to power control. The developed model is successfully validated by measurements. It is shown that the presence of power control has important implications on the achievable accuracy of the measurements. Based on the developed model, the optimal settings of the spectrum analyzer are proposed for the exposure assessment around UMTS base stations.
\end{abstract}

Index Terms - Code division multiaccess (CDMA), electromagnetic radiation, land mobile radio, spectral analysis.

\section{INTRODUCTION}

$\mathbf{P}$ EOPLE have increasingly been worrying about the possible adverse health effects of the exposure to electromagnetic radiation. These concerns have been intensified by the massive deployment of base station antennas, which are necessary to provide capacity and coverage for an ever-increasing number of subscribers and a growing demand for bandwidth. The introduction of third-generation mobile systems, which enable integrated mobile telephony and data services, implies the deployment of even more base stations and will not alleviate the problem. In answer to these questions, several authorities have issued guidelines on the maximum permissible electromagnetic field levels (e.g., [1]) to protect the general public from an excessive exposure to electromagnetic radiation. In most cases, the limits have been based on the recommendations given by international expert organizations [2], [3]. These guidelines have resulted in regulations on the installation and exploitation of electromagnetic transmitters, which have been or are being harmonized by several standardization bodies [4]-[8].

In order to check whether the present electromagnetic fields comply with the exposure limits, electromagnetic field measurements are being executed. Because the reference levels to which the measured fields have to be compared depend

Manuscript received August 15, 2006; revised March 15, 2007. The work of C. Olivier was supported by a Grant of the Fund for Scientific Research Flanders (F. W. O.-Vlaanderen).

C. Olivier is with Mobistar, 1140 Brussels, Belgium (e-mail: Christof. Olivier@telenet.be).

L. Martens is with the Department of Information Technology, Ghent University, 9000 Ghent, Belgium (e-mail: Luc.Martens@intec.UGent.be).

Digital Object Identifier 10.1109/TIM.2007.903617 on the frequency, and since the electromagnetic spectrum is densely populated with a whole range of applications, the measurements should be able to distinguish between the several emitting sources and to determine the responsible party for each exposure level. Since each application and/or operator have been assigned a separate frequency band, the use of narrowband measurements (e.g., with a spectrum analyzer) is obvious. Although for the application to signals that use code division multiaccess (CDMA), frequency-selective measurements do not provide as much information as signal analyzers, where the measured electromagnetic signal is resolved in the code domain, the general applicability of spectrum analysis to every modulated signal remains an important advantage. This is also emphasized by the introduction of new measurement equipment where portable spectrum analyzers are combined with isotropic field probes. These devices enable the quick analysis of an exposure situation, together with a characterization of the different sources.

In [9], Olivier and Martens have discussed the measurement issues that arise when mobile communication signals of the second generation (i.e., global system for mobile communications or GSM) are measured. In [10], Olivier and Martens have extended the discussion to the measurement of the wideband CDMA (WCDMA) signal, which is used in the third-generation systems [in particular for universal mobile telecommunications system (UMTS)] and developed a theoretical model for the behavior of the measured WCDMA signal for the different detector modes of the spectrum analyzer. Although power control is a very important feature of UMTS, it was not included in the model. In this paper, the theoretical model developed in [10] will be extended to a UMTS signal where power control is present. The resulting model will be validated on measurements of a generic UMTS signal (GUS). After the demonstration of the importance of power control on the achievable accuracy of the measurement, the optimal settings of the spectrum analyzer for the exposure assessment of a UMTS signal will be derived from the developed theoretical model.

\section{Influence of Power CONTROL}

In this paper, the theoretical model for the UMTS signal developed in [10] is extended to also include the effect of power control on the UMTS signal. Power control is applied to prevent the near-far problem of CDMA, where a mobile located near the base station can shout down a mobile that is far away 


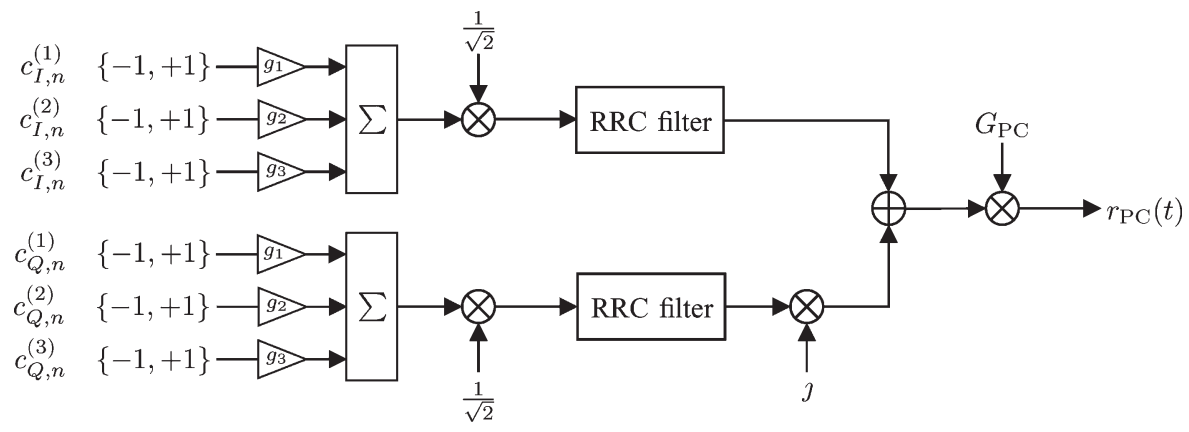

Fig. 1. Low-pass model for the UMTS signal with power control.

due to the higher path loss to which the latter is subject. To overcome this situation, the transmit power of the mobile station is adapted with a rate of 1500 times/s (corresponding to a power control period of $T_{\mathrm{PC}}=0.67 \mathrm{~ms}$ ), which is based on the quality perceived at the base station receiver. In the downlink, it is used to deliver some more power to mobiles located at the cell edge, which experience a higher interference from the neighboring cells. The power control of UMTS can even compensate the Rayleigh fading effect for slowly moving mobiles. In UMTS, the transmit power is adapted every $0.67 \mathrm{~ms}$ with a step within the range of $0.5-3 \mathrm{~dB}$. Even in the case where there is no need to change the transmit power, the power is continuously augmented and diminished with an elementary power step.

In Fig. 1, the low-pass representation for the UMTS signal with power control is given, which has been based on the representation developed in [10] for the WCDMA signal without power control. The UMTS signal is constructed from a number of independent spreading channels, and for each spreading channel $j$, a random chip stream is generated for both the in-phase $\left(c_{I, n}^{(j)}\right)$ and quadrature branch $\left(c_{Q, n}^{(j)}\right)$ of the signal at a rate of $3.84 \mathrm{MHz}$. The value of the chips is modeled to be equally probable between $\{-1,1\}$; it is assumed that subsequent chips or chips from different spreading channels are mutually independent. The in-phase and quadrature chips of each subchannel are then multiplied with their respective channel gain $g_{j}$ and summed together. The combined symbol is then sent through the pulse-shaping filter, which is a root-raised cosine filter with a roll-off factor of 0.22 . Next, the in-phase and quadrature branches are combined into a complex low-pass signal. Finally, the signal is multiplied with the power control gain $G_{\mathrm{PC}}$, which changes at a rate of $1500 \mathrm{~Hz}$. The resulting signal $r_{\mathrm{PC}}(t)$ is then the low-pass representation of the UMTS signal with power control. A more extensive description of the air interface of the UMTS system can be found in [11].

In the following, the expressions for the mean and standard deviation of the measured signal will be elaborated for the different detector modes (sample, root mean square (rms) and positive peak) of the spectrum analyzer. The signal is assumed to have a constant average power level over the long term but, on the other hand, to be subject to continuous power control. This means that the signal will subsequently switch over from a "high" to a "low" state and vice versa, which is modeled by assuming that, in the high state, the power control gain $G_{\mathrm{PC}}$ equals to 1 , and that, in the low state, the power control attenuates the signal with a factor $G_{\mathrm{PC}}=\alpha<1$ (e.g., for a step of $3 \mathrm{~dB}$, this corresponds to $\alpha=1 / \sqrt{2}$ ).

\section{A. Sample Detector}

If several measured samples can be considered as independent (this is the case if the sample period is not a multiple of $2 T_{\mathrm{PC}}$, and a sufficient number of samples are taken), the probability that the signal is in the "high" or "low" state will be equal to $1 / 2$. The probability distribution function (pdf) $f_{|S|_{\mathrm{PC}}}(s)$ of the signal with power control, which is measured by the sample detector $S_{\mathrm{PC}}$, can then be written as

$$
\begin{aligned}
f_{|S| \mathrm{PC}}(s) & =\operatorname{Pr}\left[s<|S|_{\mathrm{PC}} \leq s+d s\right] \\
& =\frac{1}{2} f_{|S|}(s)+\frac{1}{2} f_{|S|}\left(\frac{s}{\alpha}\right) \frac{1}{\alpha}
\end{aligned}
$$

where $f_{|S|}(s)$ denotes the pdf of the signal measured with the sample detector if no power control was present. In [10], it appears that this distribution is approximately a Rayleigh distribution. The mean and standard deviation can then easily be calculated as

$$
\mu_{\mathrm{smp}, \mathrm{PC}}=\frac{1+\alpha}{2} \mu_{\mathrm{smp}}
$$

and

$$
\sigma_{\mathrm{smp}, \mathrm{PC}}^{2}=\frac{1+\alpha^{2}}{2} \sigma_{\mathrm{smp}}^{2}+\frac{(1-\alpha)^{2}}{2} \mu_{\mathrm{smp}}^{2}
$$

where $\mu_{\mathrm{smp}}$ and $\sigma_{\mathrm{smp}}$ are, respectively, the mean and the standard deviation of the signal measured with the sample detector if there would have been no power control. It is clear that in the case with power control, the standard deviation on the sample signal will be much larger, which is reflected by the second term of the standard variation. If the sample time would equal an even number of power control periods, only one state of the signal would be measured, which leads to an underor overestimation of the actual power. If the sample period is chosen as an odd number of power control periods, both states will definitively be measured.

\section{B. RMS Detector}

First, the influence of power control on the mean-square (MS) signal will be examined. Assume that, during the measurement, the signal occurred during a period $T_{\mathrm{L}}$ in the low 
state, and for the rest of the measuring period, $T_{\mathrm{H}}=T_{\mathrm{S}}-T_{\mathrm{L}}$ in the high state. The measured MS signal will then be given by

$$
S_{\mathrm{MS}, \mathrm{PC}}\left(T_{\mathrm{S}}\right)=\frac{T_{\mathrm{L}}}{T_{\mathrm{S}}} S_{\mathrm{MS}, \mathrm{L}}\left(T_{\mathrm{L}}\right)+\frac{T_{\mathrm{H}}}{T_{\mathrm{S}}} S_{\mathrm{MS}, \mathrm{H}}\left(T_{\mathrm{H}}\right)
$$

where $S_{\mathrm{MS}, \mathrm{L}}\left(T_{\mathrm{L}}\right)$ and $S_{\mathrm{MS}, \mathrm{H}}\left(T_{\mathrm{H}}\right)$ are the random variables describing the MS value of the signal when it is in the low and high states, respectively. If it is assumed that both random variables are independent (which is acceptable if the inverse of the resolution bandwidth (RBW) of the spectrum analyzer is small compared to the power control period $T_{\mathrm{PC}}$ ), the characteristic function of the measured MS level, given the period that the signal is in the low state, is

$$
\begin{aligned}
\Psi_{\mathrm{MS}, \mathrm{PC} \mid T_{\mathrm{L}}}\left(u \mid T_{\mathrm{L}}\right) \\
=E\left[\exp \left(\jmath u S_{\mathrm{MS}, \mathrm{PC}}\right) \mid T_{\mathrm{L}}\right] \\
=\Psi_{\mathrm{MS}, \mathrm{H}}\left(\frac{T_{\mathrm{S}}-T_{\mathrm{L}}}{T_{\mathrm{S}}} u \mid T_{\mathrm{L}}\right) \cdot \Psi_{\mathrm{MS}, \mathrm{L}}\left(\frac{T_{\mathrm{L}}}{T_{\mathrm{S}}} u \mid T_{\mathrm{L}}\right) \\
=\exp \left(\jmath \frac{T_{\mathrm{S}}-T_{\mathrm{L}}}{T_{\mathrm{S}}} \mu_{\mathrm{H}} u\right) \\
\quad \times \exp \left(-\sigma_{\mathrm{MS}, \mathrm{H}}^{2}\left(T_{\mathrm{S}}-T_{\mathrm{L}}\right) \frac{\left(T_{\mathrm{S}}-T_{\mathrm{L}}\right)^{2}}{T_{\mathrm{S}}^{2}} u^{2}\right) \\
\quad \times \exp \left(j \frac{T_{\mathrm{L}}}{T_{\mathrm{S}}} \alpha^{2} \mu_{\mathrm{H}} u\right) \exp \left(-\alpha^{4} \sigma_{\mathrm{MS}, \mathrm{H}}^{2}\left(T_{\mathrm{L}}\right) \frac{T_{\mathrm{L}}^{2}}{T_{\mathrm{S}}^{2}} u^{2}\right)
\end{aligned}
$$

where $\mu_{\mathrm{H}}$ and $\sigma_{\mathrm{H}}$ denote the mean and standard deviation of the MS level of the signal when it is in either the high or the low state. To obtain (7), the second-order approximation of the distribution of the measured MS level of a WCDMA signal by a Gaussian distribution has been used [10], together with the relationships $\mu_{\mathrm{L}}=\alpha^{2} \mu_{\mathrm{H}}$ and $\sigma_{\mathrm{L}}=\alpha^{2} \sigma_{\mathrm{H}}$. Thus, (7) is the characteristic function of a normal distribution with mean and standard deviation given by

$$
\mu_{\mathrm{MS}, \mathrm{PC} \mid T_{\mathrm{L}}}\left(t_{\mathrm{L}}\right)=\mu_{\mathrm{MS}}\left(1+\left(\alpha^{2}-1\right) \frac{t_{\mathrm{L}}}{T_{\mathrm{S}}}\right)
$$

and

$$
\sigma_{\mathrm{MS}, \mathrm{PC} \mid T_{\mathrm{L}}}^{2}\left(t_{\mathrm{L}}\right)=\sigma_{\mathrm{MS}}^{2}\left(1+\left(\alpha^{4}-1\right) \frac{t_{\mathrm{L}}}{T_{\mathrm{S}}}\right)
$$

The mean and standard deviation of the measured MS level can be calculated if the distribution of $T_{\mathrm{L}}$, which is the period that the signal is in a low state, is known. Given a measuring period $T_{\mathrm{S}}$, the signal will approximately be in the low state during a period $N T_{\mathrm{PC}}$, where $2 N$ is the even number of power control periods closest to the measuring period

$$
N=\left[\frac{T_{\mathrm{S}}}{2 T_{\mathrm{PC}}}\right] \text {. }
$$

The operator $[\cdot]$ denotes the rounding to the closest integer. The exact length of the low period $T_{\mathrm{L}}$ will depend on the start time of the measuring period, which is assumed to be uniformly

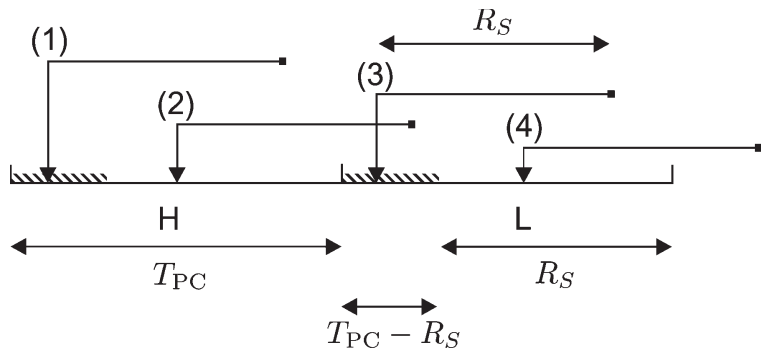

Fig. 2. Different scenarios for the beginning of the measuring periods. (1) $R_{\mathrm{S}}$ is completely located in the high state $\left[T_{\mathrm{H}}=(1 / 2)\left(T_{\mathrm{S}}+R_{\mathrm{S}}\right), T_{\mathrm{L}}=\right.$ $\left.(1 / 2)\left(T_{\mathrm{S}}-R_{\mathrm{S}}\right)\right]$. (2) and (4) The remaining interval $R_{\mathrm{S}}$ comprises both a high period and a low period $\left(T_{\mathrm{H}}=T_{\mathrm{S}}-t_{\mathrm{L}}, T_{\mathrm{L}}=t_{L}\right)$. (3) The signal is during the remainder period $R_{\mathrm{S}}$ continuously in the low state $\left[T_{\mathrm{H}}=\right.$ $\left.(1 / 2)\left(T_{\mathrm{S}}-R_{\mathrm{S}}\right), T_{\mathrm{H}}=(1 / 2)\left(T_{\mathrm{S}}+R_{\mathrm{S}}\right)\right]$.

distributed along the high and low states of the signal (i.e., an interval with length $2 T_{\mathrm{PC}}$ ).

If the measuring period is written as $T_{\mathrm{S}}=2 N T_{\mathrm{PC}}+R_{\mathrm{S}}$, and the closest even number of power control periods is smaller than $T_{S}$, the remainder part $R_{\mathrm{S}}$ will be positive and smaller than $T_{\mathrm{PC}}$. As it is indicated in Fig. 2, four situations can be distinguished.

1) The remaining part of the measurement period $R_{\mathrm{S}}$ is completely located in the high state of the signal. This situation has a probability of $\left(T_{\mathrm{PC}}-R_{\mathrm{S}}\right) /\left(2 T_{\mathrm{PC}}\right)$, which is the ratio of the shaded part of the interval to the double power control period. In this case, $T_{\mathrm{L}}$ will be equal to $N T_{\mathrm{PC}}$.

2) The remaining period $R_{\mathrm{S}}$ starts during the high state of the signal and ends in the low state. The part of the measuring period where the signal is in the low state varies between $N T_{\mathrm{PC}}$ and $N T_{\mathrm{PC}}+R_{\mathrm{S}}$.

3) The state of the signal during the entire remaining period is low; therefore, $T_{\mathrm{L}}$ will be equal to $N T_{\mathrm{PC}}+R_{\mathrm{S}}$ with a probability $\left(T_{\mathrm{PC}}-R_{\mathrm{S}}\right) /\left(2 T_{\mathrm{PC}}\right)$.

4) The period $R_{\mathrm{S}}$ starts during the low state and ends in the high state of the signal. This situation is completely analogous to the second case.

To summarize, the pdf of $T_{\mathrm{L}}$ is given by

$$
\operatorname{Pr}\left[T_{\mathrm{L}}=\frac{T_{\mathrm{S}}-R_{\mathrm{S}}}{2}\right]=\frac{T_{\mathrm{PC}}-R_{\mathrm{S}}}{2 T_{\mathrm{PC}}}
$$

Case (1)

$$
\begin{aligned}
& \operatorname{Pr}\left[t_{\mathrm{L}}<T_{\mathrm{L}} \leq t_{\mathrm{L}}+d t_{\mathrm{L}}\right] \\
& =\frac{1}{T_{\mathrm{PC}}} d t_{\mathrm{L}}, \quad T_{\mathrm{L}} \in\left[\frac{T_{\mathrm{S}}-R_{\mathrm{S}}}{2}, \frac{T_{\mathrm{S}}+R_{\mathrm{S}}}{2}\right] \\
& \text { Case }(2) \text { and }(4) \\
& \operatorname{Pr}\left[T_{\mathrm{L}}=\frac{T_{\mathrm{S}}+R_{\mathrm{S}}}{2}\right]=\frac{T_{\mathrm{PC}}-R_{\mathrm{S}}}{2 T_{\mathrm{PC}}}
\end{aligned}
$$

Case (3).

If the closest even number of power control periods is larger than the measuring period $T_{\mathrm{S}}$, this period can be written as $T_{\mathrm{S}}=2 N T_{\mathrm{PC}}-R_{\mathrm{S}}$, where $0 \leq R_{\mathrm{S}}<T_{\mathrm{PC}}$. Following an 
analogous argumentation as above, it can be shown that the distribution of $T_{\mathrm{L}}$ is, in this case, also given by (11). Therefore, by defining $R_{\mathrm{S}}$ as

$$
R_{\mathrm{S}}=\left|T_{\mathrm{S}}-2 T_{\mathrm{PC}} N\right|
$$

and with $N$ defined by (10), (11) provides a general distribution of the part of the measuring period that the signal was in a low state.

Knowing this distribution, the mean and standard deviation of the measured MS signal for the signal with power control can easily be derived from (8), (9), and (11) as

$$
\begin{aligned}
\mu_{\mathrm{MS}, \mathrm{PC}}= & \frac{1+\alpha^{2}}{2} \mu_{\mathrm{MS}} \\
\sigma_{\mathrm{MS}, \mathrm{PC}}= & \frac{1+\alpha^{4}}{2} \sigma_{\mathrm{MS}}^{2}+\frac{\left(1-\alpha^{2}\right)^{2}}{4} \\
& \times\left(1-\frac{2}{3} \frac{R_{\mathrm{S}}}{T_{\mathrm{PC}}}\right)\left(\frac{R_{\mathrm{S}}}{T_{\mathrm{S}}}\right)^{2} \mu_{\mathrm{MS}}^{2} .
\end{aligned}
$$

The standard deviation shows an important dependence on the length of the measuring interval. It is also clear that the MS signal will show minimal variation whenever the measuring period is an even number of power control intervals, since, in that case, the signal is during an exactly equal portion of the measuring time in the high and low states, respectively.

The pdf of the measured rms signal of a WCDMA signal with power control can be derived directly by integrating the dependence of the pdf on the low period $t_{\mathrm{L}}$ as

$$
\begin{aligned}
f_{\mathrm{RMS}, \mathrm{PC}}(r)= & \int_{\frac{1}{2}\left(T_{\mathrm{S}}-R_{\mathrm{S}}\right)}^{\frac{1}{2}\left(T_{\mathrm{S}}+R_{\mathrm{S}}\right)} f_{T_{\mathrm{L}}}\left(t_{\mathrm{L}}\right) \sqrt{\frac{2}{\pi}} \frac{r}{\sigma_{\mathrm{MS}, \mathrm{PC} \mid \mathrm{T}_{\mathrm{L}}}\left(t_{\mathrm{L}}\right)} \\
& \times \exp \left(-\frac{\left(r^{2}-\mu_{\mathrm{MS}, \mathrm{PC} \mid \mathrm{T}_{\mathrm{L}}}\left(t_{\mathrm{L}}\right)\right)^{2}}{2 \sigma_{\mathrm{MS}, \mathrm{PC} \mid \mathrm{T}_{\mathrm{L}}}\left(t_{\mathrm{L}}\right)}\right) d t_{\mathrm{L}}
\end{aligned}
$$

where $\mu_{\mathrm{MS}, \mathrm{PC} \mid \mathrm{T}_{\mathrm{L}}}\left(t_{\mathrm{L}}\right)$ and $\sigma_{\mathrm{MS}, \mathrm{PC} \mid \mathrm{T}_{\mathrm{L}}}\left(t_{\mathrm{L}}\right)$ are the mean and standard deviation, respectively, of the measured MS signal, given the low period $t_{\mathrm{L}}$, which are defined by (8) and (9); $f_{T_{\mathrm{L}}}\left(t_{\mathrm{L}}\right)$ denotes the pdf of the part of the measuring period where the signal is low and is defined by (11). This distribution can numerically be calculated, from which the mean and standard deviation of the rms signal can also be derived. To obtain (15), the fact that the MS distribution can be approximated by a Gaussian function (7) and that the rms distribution is related to the pdf of the MS signal by $f_{\mathrm{RMS}}(r)=2 r f_{\mathrm{MS}}\left(r^{2}\right)$ has been used.

To verify the model developed in [10], the predicted results have been compared to measurements of a GUS, as described in [12]. The GUS has been developed for biological experiments, and it mimics the worst-case power behavior of the UMTS signal. The GUS signal generator has been chosen to validate the theoretical model because it produces a repetitive signal while representing the characteristics of a realistic UMTS signal (spread spectrum through CDMA, power control, configured according to the specifications). To model the effects of
TABLE I

Comparison Between the Measured and Predicted Mean RMS VALUES AS A FunCTION OF THE RBW FOR A MEASUREMENT PERIOD $T_{\mathrm{S}}$ OF $1.3 \mathrm{~ms}$

\begin{tabular}{l|cc}
\hline RBW & $\begin{array}{c}\mu_{\text {RMS,PC,meas }} \\
{[\mathrm{mV}]}\end{array}$ & $\begin{array}{c}\mu_{\mathrm{RMS}, \mathrm{PC}, \mathrm{calc}} \\
{[\mathrm{mV}]}\end{array}$ \\
\hline $10 \mathrm{kHz}$ & 7.6 & 7.2 \\
$100 \mathrm{kHz}$ & 24 & 23 \\
$300 \mathrm{kHz}$ & 55 & 51 \\
$1 \mathrm{MHz}$ & 77 & 73 \\
$5 \mathrm{MHz}$ & 131 & 163 \\
\hline
\end{tabular}

power control on the signal, the GUS signal generator produces during one period (approximately $45 \mathrm{~s}$ ) a signal with a constant average level, while during the next period, the power of the generated signal is continuously adapted to mimic strong fading conditions. For the measurements considered here, the GUS signal was only measured in the period where the (average) transmitted power was kept constant. For the GUS signal, this means that the power is continuously adapted with steps $\pm 3 \mathrm{~dB}$ (to represent a worst case scenario). It should also be mentioned that the power of the GUS signal showed a small slowly changing variation of $0.15 \mathrm{~dB}$.

As it appears from (13), the expected MS value will show no dependence on the length of the measuring period. The expected rms value will neither show a significant dependence on the chosen measurement time. In Table I, the comparison is made up of the mean rms levels that are measured on the GUS signal and the mean rms levels that are predicted by the theoretical model when the measurement period $T_{\mathrm{S}}$ is chosen as $1.3 \mathrm{~ms}$. There is a good agreement between the predicted and measured mean rms values, except for the RBW of $5 \mathrm{MHz}$, where the theoretical model is no longer valid. Indeed, in [10], it was assumed that the resolution filter was located entirely within the flat frequency part of the WCDMA pulseshaping filter, and this assumption is no longer valid for a RBW of $5 \mathrm{MHz}$, as illustrated in Fig. 3. Because, in the theoretical model, it is assumed that the UMTS signal has a constant power density over the entire width of the resolution filter, the predicted levels for the $5-\mathrm{MHz}$ filter will be higher than the actual measured levels.

The standard deviation on the measured rms level, in contrast with its mean value, will depend on the length of the measuring period, as it is shown in Fig. 4 for the measured rms level of the GUS signal. The comparison is also made between the standard deviation on the measured rms levels of the GUS signal and the standard deviation predicted through (15). As could be expected from (14), the standard deviation is smallest if the measuring period contains an even number of power control periods. If the measuring period is an odd number of power control periods, the standard deviation shows a local maximum. Except for the large RBW of $5 \mathrm{MHz}$, the agreement between simulations and measurements is excellent. For $5 \mathrm{MHz}$, the deviation is due to the false assumption that the resolution filter of the spectrum 


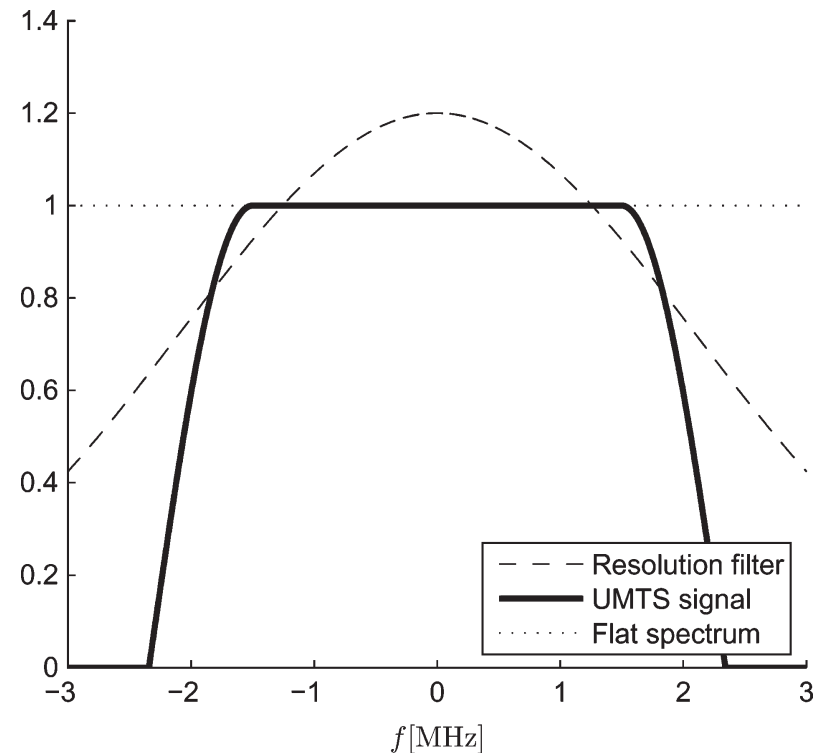

Fig. 3. Approximation of the 5-MHz wide UMTS signal by a flat spectrum signal. The 5-MHz wide resolution filter is given as a reference.

analyzer is located completely within the flat frequency part of the WCDMA pulse-shaping filter. It is also shown that, for measuring periods $T_{\mathrm{S}}<2 T_{\mathrm{PC}}$, the standard deviation on the rms value is mainly caused by the power variation of the measured signal. For the small RBW of $10 \mathrm{kHz}$, the effect of the power control on the standard deviation is less obvious since the relative standard deviation inherent to the use of a small resolution filter is much larger. In Fig. 4, it also appears that the standard deviation of the rms measurement decreases with the increasing measuring period, and if the effect of power control can be neglected, the standard deviation decreases following the law $1 / \sqrt{T_{\mathrm{S}}}$. In the model, the small slowly changing power variation of the GUS signal has also be included and was supposed uniformly distributed. The variation of the power causes the decreasing trend of the standard deviation to deflect at a certain level of standard deviation, since there remains a minimum standard deviation due to this slow power variation of the GUS signal.

\section{Positive-Peak Detector}

Given the part of the measuring period $T_{\mathrm{S}}$ where the signal is low $T_{\mathrm{L}}$, the cumulative distribution derived in [10] can be extended for power control to

$$
\begin{aligned}
& F_{M, \mathrm{PC} \mid T_{\mathrm{L}}}\left(m \mid t_{\mathrm{L}}\right) \\
& =\operatorname{Pr}\left[\max _{0 \leq t<t_{\mathrm{L}}} \alpha|s(t)|<m\right] \cdot \operatorname{Pr}\left[\max _{0 \leq t<T_{\mathrm{S}}-t_{\mathrm{L}}}|s(t)|<m\right] \\
& =\left(1-\frac{\bar{\nu}(m / \alpha)}{\bar{\nu}(0)}\right)^{\bar{\nu}(0) t_{\mathrm{L}}} \cdot\left(1-\frac{\bar{\nu}(m)}{\bar{\nu}(0)}\right)^{\bar{\nu}(0)\left(T_{\mathrm{S}}-t_{\mathrm{L}}\right)}
\end{aligned}
$$

where $\bar{\nu}(m)$ denotes the average number of maxima within a period of $\sigma_{t}$, lying above the level $m$, where levels are normalized to the square root of the expected MS level [10]. The pdf of the part of the measuring period that the signal is

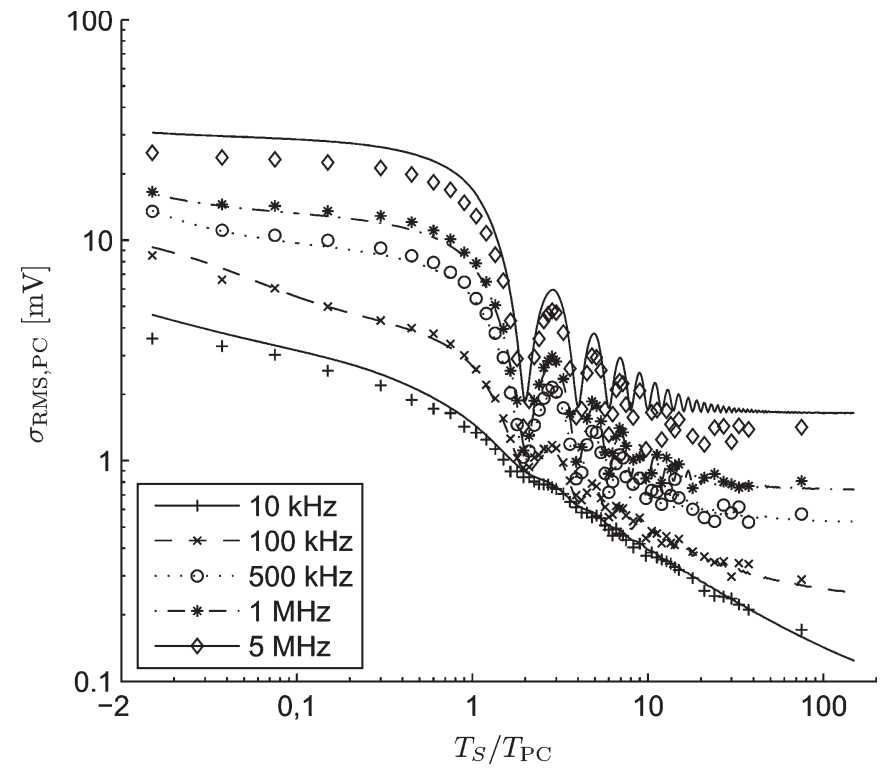

Fig. 4. Comparison between the standard deviation on the measured rms signal (indicated with markers) of the GUS signal and the standard deviation calculated from the pdf (15) (indicated with lines) and its dependence on the duration of the measurement period $T_{\mathrm{S}}$. The standard deviation is indicated for different RBWs $(10 \mathrm{kHz}, 100 \mathrm{kHz}, 500 \mathrm{kHz}, 1 \mathrm{MHz}$, and $5 \mathrm{MHz}$ ).

low $T_{\mathrm{L}}$ is given by (11). Given this distribution, the general cumulative distribution of the measured positive-peak signal with power control can be calculated as

$$
\begin{aligned}
F_{M, \mathrm{PC}}(m) & \int_{\frac{1}{2}\left(T_{\mathrm{S}}-R_{\mathrm{S}}\right)} f_{T_{\mathrm{L}}}\left(t_{\mathrm{L}}\right) F_{M, \mathrm{PC} \mid T_{\mathrm{L}}}\left(m \mid t_{\mathrm{L}}\right) d t_{\mathrm{L}} \\
= & \frac{T_{\mathrm{PC}}-R_{\mathrm{S}}}{2 T_{\mathrm{PC}}} \\
& \times\left(\left(1-\frac{\bar{\nu}(m / \alpha)}{\bar{\nu}(0)}\right)^{\bar{\nu}(0) \frac{T_{\mathrm{S}}+R_{\mathrm{S}}}{2}}\left(1-\frac{\bar{\nu}(m)}{\bar{\nu}(0)}\right)^{\bar{\nu}(0) \frac{T_{\mathrm{S}}-R_{\mathrm{S}}}{2}}\right. \\
& \left.\quad+\left(1-\frac{\bar{\nu}(m / \alpha)}{\bar{\nu}(0)}\right)^{\bar{\nu}(0) \frac{T_{\mathrm{S}}-R_{\mathrm{S}}}{2}}\left(1-\frac{\bar{\nu}(m)}{\bar{\nu}(0)}\right)^{\bar{\nu}(0) \frac{T_{\mathrm{S}}+R_{\mathrm{S}}}{2}}\right) \\
& +\frac{1}{\bar{\nu}(0) T_{\mathrm{PC}} \ln \left(\frac{\bar{\nu}(0)-\bar{\nu}(m / \alpha)}{\bar{\nu}(0)-\bar{\nu}(m)}\right)} \\
& \times\left(\left(1-\frac{\bar{\nu}(m / \alpha)}{\bar{\nu}(0)}\right)^{\bar{\nu}(0) \frac{T_{\mathrm{S}}+R_{\mathrm{S}}}{2}}\left(1-\frac{\bar{\nu}(m)}{\bar{\nu}(0)}\right)^{\bar{\nu}(0) \frac{T_{\mathrm{S}}-R_{\mathrm{S}}}{2}}\right. \\
& \left.\quad-\left(1-\frac{\bar{\nu}(m / \alpha)}{\bar{\nu}(0)}\right)^{\bar{\nu}(0) \frac{T_{\mathrm{S}}-R_{\mathrm{S}}}{2}}\left(1-\frac{\bar{\nu}(m)}{\bar{\nu}(0)}\right)^{\bar{\nu}(0) \frac{T_{\mathrm{S}}+R_{\mathrm{S}}}{2}}\right) .
\end{aligned}
$$

Once the cumulative distribution function (cdf) of the signal with power control is known, the pdf can be easily numerically derived from (19). 


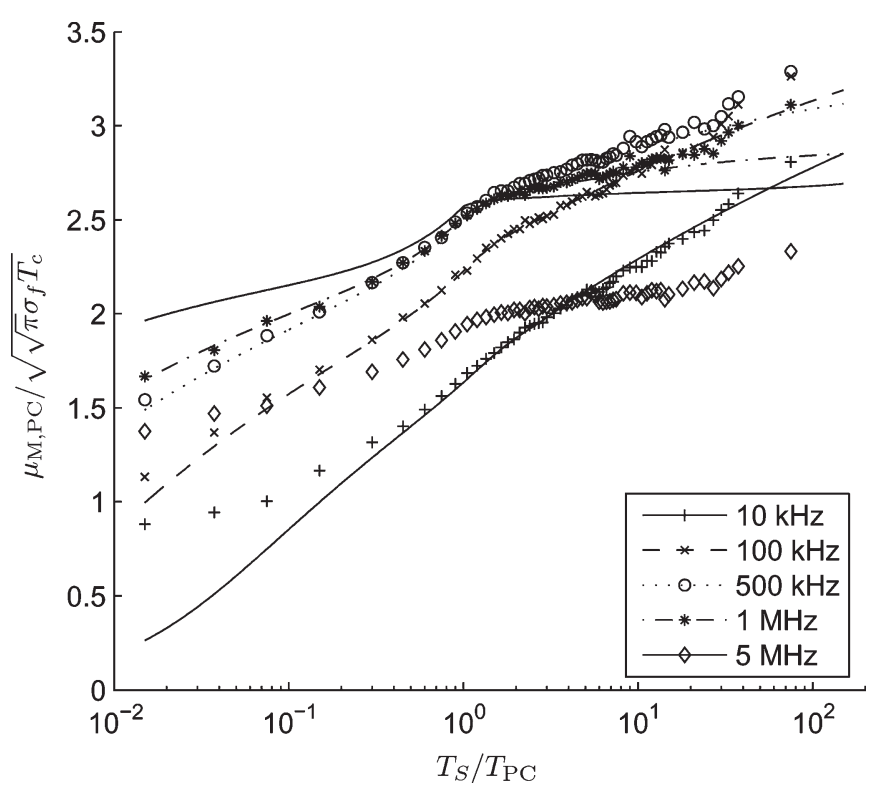

Fig. 5. Comparison between the mean of the normalized measured positivepeak signal (indicated with markers) of the GUS signal and the mean calculated from the cdf (19) (indicated with lines) and its dependence on the duration of the measurement period $T_{\mathrm{S}}$. The mean is indicated for different RBWs $(10 \mathrm{kHz}$, $100 \mathrm{kHz}, 500 \mathrm{kHz}, 1 \mathrm{MHz}$, and $5 \mathrm{MHz}$ ).

Again, the predicted mean and standard deviation of the distribution of the positive-peak signal have been compared with the mean and standard deviation of the positive-peak measurements on the GUS signal (see Figs. 5 and 6, respectively). Both the mean and standard deviation, analogous to the study in [10], have been normalized to the square root of the expected MS value of the UMTS signal if it is in its "high" state. For the predicted values, the fourth-order approximation has been applied to calculate the average rate of maxima $\bar{\nu}(m)$ above a normalized level $m$. It should be noted that this fourth-order rate $\bar{\nu}(m)$ depends on the difference between the observation frequency at which the signal is measured and the carrier frequency, on the RBW of the spectrum analyzer and on the number of parallel channels transmitted.

As it is indicated in Fig. 5, the expected value of the level returned by the positive-peak detector increases with increasing measuring periods $T_{\mathrm{S}}$. The predicted mean shows a good agreement with the mean of the measurements for the different RBWs (except for the 5-MHz resolution filter, because then, the pulse-shaping filter cannot be considered as flat over the width of the resolution filter). Although, for the RBW of $5 \mathrm{MHz}$, the model is not valid, the evolution with respect to the measuring period $T_{\mathrm{S}}$ shows the same variation. There is also a deviation for the $10-\mathrm{kHz}$ RBW for short measuring times $T_{\mathrm{S}}$ due to the long time response of the small resolution filter (otherwise stated, because the sweep rate, which is defined as the ratio of the frequency span to the sweep time, was too high and did not satisfy the condition mentioned in [13]). In Fig. 5, it can also be observed that the curve shows a breaking point around the power control period $T_{\mathrm{PC}}$. This could be expected since, for longer measuring periods, the distribution of the mean will be dominated by the period where the signal was in the high state, while for smaller measuring periods, both the distributions of

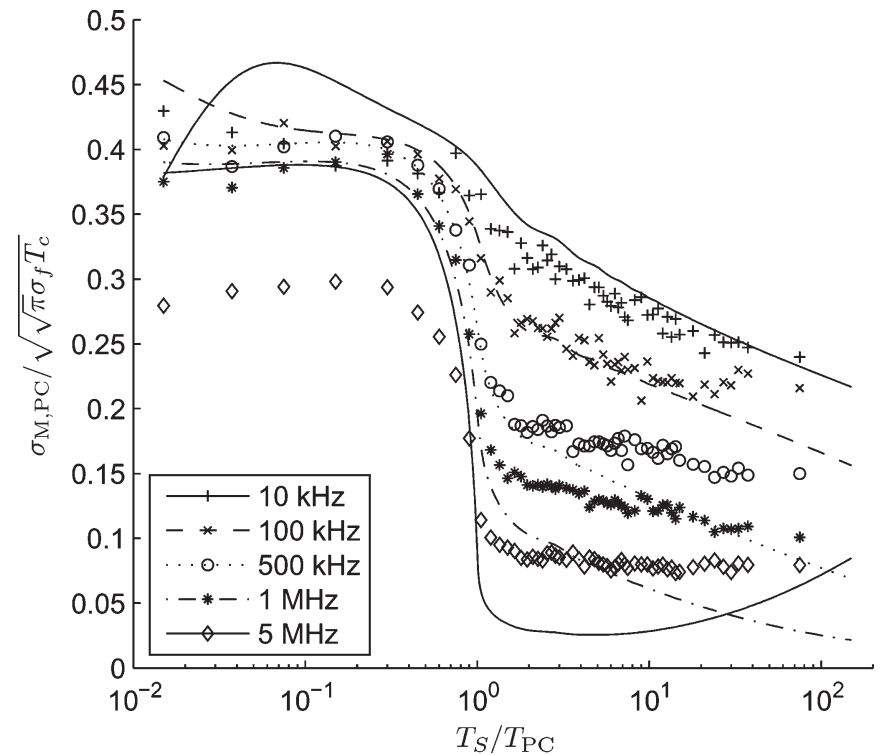

Fig. 6. Comparison between the standard deviation on the normalized measured positive-peak signal (indicated with markers) of the GUS signal and the standard deviation calculated from the cdf (19) (indicated with lines) and its dependence on the duration of the measurement period $T_{\mathrm{S}}$. The standard deviation is indicated for different RBWs $(10 \mathrm{kHz}, 100 \mathrm{kHz}, 500 \mathrm{kHz}, 1 \mathrm{MHz}$, and $5 \mathrm{MHz}$ ).

the signal in the high as in the low state will contribute to the total distribution.

As shown in Fig. 6, the standard deviation on the positivepeak signal is for measurement periods $T_{\mathrm{S}}$ smaller than $T_{\mathrm{PC}}$, which is dominated by the variation due to power control and remains almost constant. For longer measurement periods, the positive-peak level is dominated by the high state of the signal, and accordingly, the standard deviation on the positive-peak level decreases for longer measurement periods, as predicted in [10]. The agreement between the measurements is rather good for the $10-$ and $100-\mathrm{kHz}$ resolution filter and somewhat worse for the $1-\mathrm{MHz}$ resolution filter. As it could be expected, the predicted standard deviation on the positivepeak signal that is measured with the $5-\mathrm{MHz}$ resolution filter does not agree with the standard deviation of the measurements because of the invalidity of the theoretical model. However, for both the $1 \mathrm{MHz}$ as the $5-\mathrm{MHz}$ resolution filter, the main trend of the standard deviation is well predicted.

\section{Optimal Settings of the Spectrum AnAlyzer}

\section{A. Goal of the Measurements}

If the UMTS signal is measured to assess the exposure on a certain location, the goal of the measurement is to determine the maximum electric or magnetic field to which a person, located at the measurement position, might be exposed. Therefore, the characterization should ideally identify the maximum electric or magnetic field level that could occur in time. In contrast with code analyzers, the spectrum analyzer cannot distill the power of the pilot channel out of the UMTS signal to estimate this maximum field level. However, this maximum may be estimated from the maximum electromagnetic field level that has occurred during the measuring time, in analogy with 


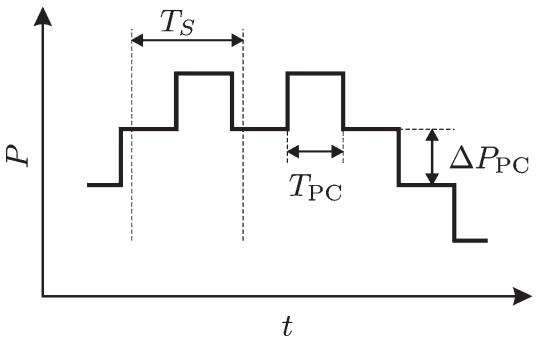

Fig. 7. Optimal choice of the duration of the measurement period $T_{\mathrm{S}}$ for a UMTS signal (one channel) with power control.

methods using a broadband field probe to assess the exposure [14]. The choice of settings of the spectrum analyzer should, therefore, be chosen carefully to obtain a minimum uncertainty on the estimated maximum field level.

\section{B. Choice of the Measuring Period}

As it was demonstrated in [10], the standard deviation on the measurement of a WCDMA signal decreases with longer measurement periods, both for the rms detector as for the positivepeak detector; hence, the measuring period should be chosen as long as possible. However, since, in UMTS, tight power control is used, the fast variations (every $0.67 \mathrm{~ms}$ ) due to power control will provide a limitation on the maximum measuring period. Consider a signal where one spreading channel is dominant (see Fig. 7): if the measurement period is longer than $2 T_{\mathrm{PC}}$, it is impossible to state from the measured maximum whether this maximum was due to the occurrence of a single maximum power level or due to several subsequent maxima. For the rms detector, this would mean that, if the maximum power was transmitted, it is not certain whether the maximum possible rms level was measured. For the positive-peak detector, the situation is even worse: since the ratio between the measured positivepeak level and the actual rms level depends on the time that the signal was in its maximum state, an accurate estimate of the rms level cannot be made if this time is not known. Another way to alleviate this problem is by choosing the measurement period that is shorter than the half power control period. Then, the maximum rms level will certainly be detected, and it could also be estimated from the positive-peak measurement. However, a shorter measurement time corresponds to a larger standard deviation on the measurements.

Ideally, the measuring period should, thus, be chosen equally to $2 T_{\mathrm{PC}}$. If one sweep of the spectrum analyzer contains $N_{\mathrm{bin}}$ measuring points, then the total sweep time over the $N_{\text {bin }}$ frequency bins should be set to $N_{\text {bin }} \times 2 T_{\mathrm{PC}}$. For a spectrum analyzer displaying, e.g., 500 measurement points on its screen, this corresponds to a sweep time of $667 \mathrm{~ms}$. Shorter sweep times are allowable at the cost of larger uncertainty, provided that the measurement period is shorter than half the power control period. This corresponds to a measuring period of $N_{\text {bin }} T_{\mathrm{PC}} / 2$ or, for 500 frequency bins, a sweep time smaller than $167 \mathrm{~ms}$. To estimate the worst case exposure level, the maximum over the whole bandwidth of the UMTS signal should be determined. For the rms detector, this maximum level is the sought value, while for the positive-peak detector, the

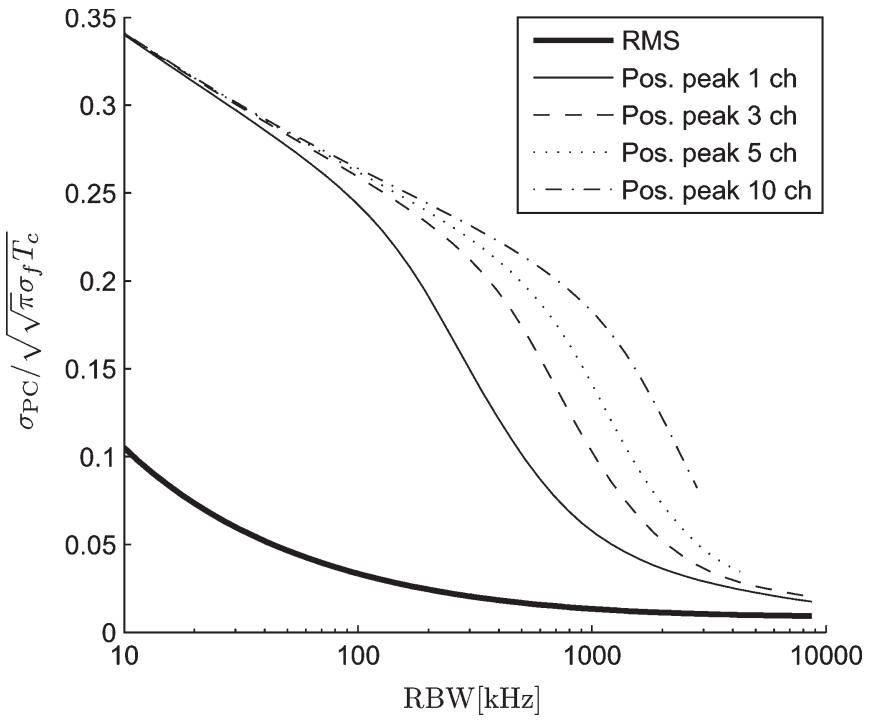

Fig. 8. Variation of the standard deviation $\sigma_{\mathrm{PC}}$ on the measured level for the rms (thick solid line) and the positive-peak detector (thin lines), normalized to the square root of the expected MS level, as a function of the RBW and for different numbers of transmitting channels. The measuring period $T_{\mathrm{S}}$ was chosen as $2 T_{\mathrm{PC}}$.

maximum rms value has to be extrapolated from the maximum positive-peak level. It is obvious that the measured or estimated rms level of the signal has to be corrected for the width of the resolution filter by dividing the field value with the square root of the expected MS level, $\sqrt{\sqrt{\pi} \sigma_{f} T_{c}}$ (see also [10]).

\section{Resolution Filter}

From the pdf for the measured rms level of the UMTS signal with power control (15) and the cdf for the measured positivepeak level (19), the dependence of the standard deviation of the measured signal level on the RBW can be calculated, which is shown in Fig. 8. It is clear that the relative standard deviation decreases as the RBW increases. Indeed, the time response of a wide resolution filter will be more restricted in time compared to a narrow resolution filter, and hence, less subsequent chips will contribute to the measurement result. Accordingly, the variation on the measurement result will be smaller for large-resolution filters. It should be noted that, since the level measured by the positive-peak detector is larger than the rms level, the relative standard deviation for the positivepeak detector would be smaller if it would be normalized to the measured positive-peak level instead to the square root of the MS level. Depending on the number of spreading channels present, the standard deviation on the measured positive-peak level shows a breakpoint for a certain width of the resolution filter, after which, the curve decreases more steeply. Because the signal measured by the spectrum analyzer shows a more discrete behavior for large-RBWs, and because the measured level with the positive-peak detector depends on the extremities of the pdf of the measured level [10], the standard deviation on the UMTS signal measured with the positive-peak detector is strongly reduced for larger RBWs. The breakpoint shifts toward larger RBWs if there are more equally strong spreading channels present. Accordingly, the standard deviation on the 
level measured with the positive-peak detector increases for multiple channels.

Although, in Fig. 8, it could be concluded that a larger resolution filter is advantageous, the situation where two neighboring channels are present should also be considered. If a wide resolution filter would be used, the contribution of a strong neighboring channel could lead to the overestimation of the power in the considered channel (see also the discussion in [9]) and, thus, a degraded resolution quality. In [10], it was shown that, in the situation with one dominant channel, the pdf of the signal level measured by the positive-peak detector has a mixed discrete-continuous behavior for RBWs larger than $500 \mathrm{kHz}$. This threshold between the continuous and discrete character of the distribution also depends on the number of dominating channels, and thus, it is advantageous to limit the resolution filter up to the smallest threshold for the discrete behavior, i.e., $500 \mathrm{kHz}$. Another side effect of the smaller resolution filter is the larger number of measurement points that are located in the flat frequency part of the resolution filter, which increases the chance to detect a maximum.

\section{Multiple Channels}

As shown in Fig. 5, the expected value of the measured positive-peak signal shows a fixed relationship to the rms level of the signal, depending on the chosen RBW, the measuring time. This implies that the rms power level of the signal can be estimated from the positive-peak measurements, which might be useful in situations where there is no rms detector present at the spectrum analyzer. This relationship between the positivepeak measured level and the rms level will also depend on the number of dominating channels within the UMTS signal, as shown in Fig. 9, where the curves of the ratio between the positive-peak level and the normalized rms level are given as a function of the RBW and for different numbers of transmitting channels. Since the objective of exposure assessment around base stations is to determine the worst-case exposure situation, and because for an in situ measurement the number of dominating channels is not known, the minimum ratio between the level measured by the positive-peak detector and the rms level of the signal for all channel configurations should be used in order to extrapolate the worst case maximum rms level from a positive-peak measurement. In Fig. 9, it is clear that the situation where only one channel dominates the signal represents the worst case. In Fig. 9, the curve representing the ratio to be used to extrapolate the maximum rms level of the UMTS signal from the positive-peak level is also indicated with a bold line. For small RBWs $(<200 \mathrm{kHz})$, it follows the ratio for the one channel case, and for larger RBWs, it is limited to a factor of 2.5 .

\section{E. Summary}

According to the discussion in the previous paragraphs, the proposed optimal settings for the measurement of the maximum rms signal level of a UMTS signal are summarized in Table II.

To validate these optimal settings, the maximum rms level of the GUS signal, as described in [12], has been estimated

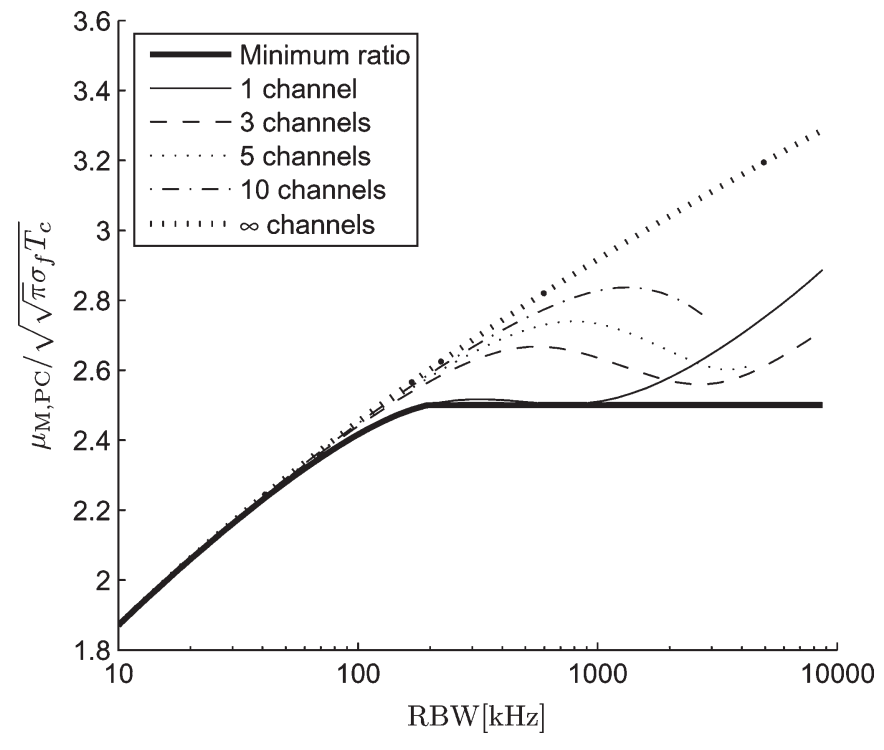

Fig. 9. Variation of the ratio of the expected level measured by the positivepeak detector and the square root of the expected MS level as a function of the RBW and for different numbers of transmitting channels. The measuring period $T_{\mathrm{S}}$ was chosen as $2 T_{\mathrm{PC}}$. The curve, which is used to estimate the maximum rms level of the signal from a positive-peak measurement, is indicated by a thick solid line.

TABLE II

Proposed SPECTRUm ANALYZER SetTings

\begin{tabular}{lc|c}
\hline Measurement period & $T_{s}$ & $1.33 \mathrm{~ms}$ \\
Resolution bandwidth & RBW & $500 \mathrm{kHz}$ \\
Detector & & RMS \\
Ratio pos. peak to RMS & & 2.5 \\
\hline
\end{tabular}

from the measurements, while the signal was subject to strong power variations. The sweep time of the spectrum analyzer was set to $680 \mathrm{~ms}$, and different resolution filters have been used $(100 \mathrm{kHz}, 300 \mathrm{kHz}, 500 \mathrm{kHz}$, and $1 \mathrm{MHz})$. For the different RBWs, the measured field level was corrected for the use of a small resolution filter. For the positive-peak detector, the rms level was extrapolated from the measured level using Fig. 9. The results from these measurements are given in Table III, where the maximum rms level measured by a resolution filter of $10 \mathrm{MHz}$ containing the whole WCDMA channel is also indicated as a reference. To obtain these figures, the maxima of the positive-peak trace and of the rms trace during one sweep were retained. Since, for the signal under test, the signal reached its maximum level several times during one sweep, the retained maximum will be determined by the upper tail of its probability distribution, and therefore, the maximum will be higher if the standard deviation on the measured level is higher. Moreover, the curve, which is used to extrapolate the maximum rms level from the maximum measured signal with the positive-peak detector, represents the worst case where only one spreading channel is dominant, while the GUS signal contains three spreading channels. This explains why, in Table III, the extrapolated levels are higher than the sought value for smaller resolution filters and why the level extrapolated from 
TABLE III

MAXimum RMS LEVEl (MEAN AND STANDARD DEVIATION) DETERMINED BY THE RMS DETECTOR OR ESTIMATED FROM Measurements With the Positive-PeAK Detector FOR DIFFERENT RESOLUTION FILTERS

\begin{tabular}{l|cc|cc}
\hline RBW & $\begin{array}{c}\mu_{\mathrm{RMS}, \mathrm{RMS}} \\
{[\mathrm{mV}]}\end{array}$ & $\begin{array}{c}\sigma_{\mathrm{RMS}, \mathrm{RMS}} \\
{[\mathrm{mV}]}\end{array}$ & $\begin{array}{c}\mu_{\mathrm{RMS}, \text { pos }} \\
{[\mathrm{mV}]}\end{array}$ & $\begin{array}{c}\sigma_{\mathrm{RMS}, \text { pos }} \\
{[\mathrm{mV}]}\end{array}$ \\
\hline $100 \mathrm{kHz}$ & 168 & 4 & 208 & 12 \\
$300 \mathrm{kHz}$ & 162 & 3 & 202 & 11 \\
$500 \mathrm{kHz}$ & 161 & 3 & 201 & 9 \\
$1 \mathrm{MHz}$ & 160 & 2 & 190 & 7 \\
\hline $10 \mathrm{MHz}$ (reference) & 141 & 1 & - & - \\
\hline
\end{tabular}

the positive-peak detector shows the largest deviation from the maximum rms level.

\section{CONCLUSION}

In this paper, the authors have extended the theoretical model for the UMTS signal measured by a spectrum analyzer for the case where the signal is subject to power control. The developed model has been validated on measurements, and from the observed behavior, the optimal settings for the spectrum analyzer for the measurement of a UMTS signal have been derived. Although the rms detector is preferred for the estimation of the maximum rms level of a UMTS signal, it is also possible to estimate this level from a measurement with a positive-peak detector, provided that an appropriate correction to the measured level, which is due to the noiselike behavior of the WCDMA signal, is made. For an optimal assessment of the maximum rms level of the UMTS signal, the measurement period over one frequency bin should be equal to two times the power control period, and the resolution filter should be chosen as $500 \mathrm{kHz}$.

\section{REFERENCES}

[1] European Council, "Council recommendation of 12 July 1999 on the limitation of exposure of the general public to electromagnetic fields (0 Hz-300 GHz)," Off. J. Eur. Communities, no. L199, pp. 59-70, 1999.

[2] International Commission on Non-Ionizing Radiation Protection (ICNIRP), "Guidelines for limiting exposure to time-varying electric, magnetic, and electromagnetic fields (up to $300 \mathrm{GHz}$ )," Health Phys., vol. 74, no. 4, pp. 494-594, Apr. 1998.

[3] IEEE Standard for Safety Levels With Respect to Human Exposure to Radio Frequency Electromagnetic Fields, $3 \mathrm{kHz}$ to $300 \mathrm{GHz}$, IEEE Std. C95.1-1991, 1999.

[4] CLC/TC106X, Basic Standard for the Calculation and Measurement of Electromagnetic Field Strength and SAR Related to Human Exposure From Radio Base Stations and Fixed Terminal Stations for Wireless Telecommunication Systems $(110 \mathrm{MHz}-40 \mathrm{GHz})$, CENELEC Std. EN 50 383:2002, 2002.

[5] CLC/TC106X, Product Standard to Demonstrate the Compliance of Radio Base Stations and Fixed Terminal Stations for Wireless Telecommunication Systems With the Basic Restrictions or the Reference Levels Related to Human Exposure to Radio Frequency Electromagnetic Fields (110 MHz-40 GHz)-Occupational, CENELEC Std. EN 50 384:2002, 2002.

[6] CLC/TC106X, Product Standard to Demonstrate the Compliance of Radio Base Stations and Fixed Terminal Stations for Wireless Telecommunication Systems With the Basic Restrictions or the Reference Levels Related to Human Exposure to Radio Frequency Electromagnetic Fields
(110 MHz-40 GHz)—General Public, CENELEC Std. EN 50 385:2002, 2002.

[7] CLC/TC106X, Basic Standard for the Calculation and Measurement of Electromagnetic Fields Related to Human Exposure From Radio Base Stations and Fixed Terminal Stations for Wireless Telecommunication Systems (110 MHz-40 GHz), When Put Into Service, CENELEC Std. prEN 50 400:2004, 2004.

[8] CLC/TC106X, Product Standard to Demonstrate the Compliance of Radio Base Stations and Fixed Terminal Stations for Wireless Telecommunication Systems With the Basic Restrictions or the Reference Levels Related to General Public Human Exposure to Radio Frequency Electromagnetic Fields (110 MHz-40 GHz), When Put Into Service, CENELEC Std. prEN 50 401:2004, 2003.

[9] C. Olivier and L. Martens, "Optimal settings for narrow band signal measurements used for exposure assessment around GSM base station antennas," IEEE Trans. Instrum. Meas., vol. 54, no. 1, pp. 311-317, Feb. 2005.

[10] C. Olivier and L. Martens, "Theoretical derivation of the stochastic behavior of a WCDMA signal measured with a spectrum analyzer," IEEE Trans. Instrum. Meas., vol. 55, no. 2, pp. 603-614, Apr. 2006.

[11] H. Holma and A. Toskala, Eds., WCDMA for UMTS. West Sussex, U.K.: Wiley, 2000.

[12] N. Ndoumbè Mbonjo Mbonjo, J. Streckert, A. Bitz, V. Hansen, A. Glasmachers, S. Gencol, and D. Rozic, "Generic UMTS test signal for RF bioelectromagnetic studies," Bioelectromagn., vol. 25, no. 6, pp. 415425, Sep. 2004.

[13] C. Rauscher, Fundamentals of Spectrum Analysis. München, Germany: Rohde and Schwarz, 2001.

[14] A. Silvi, A. Zari, and G. Licitra, "Assessment of the temporal trend of the exposure of people to electromagnetic fields produced by base stations for mobile telephones," J. Radiat. Prot. Dosimetry, vol. 97, no. 4, pp. 387 390, 2001.

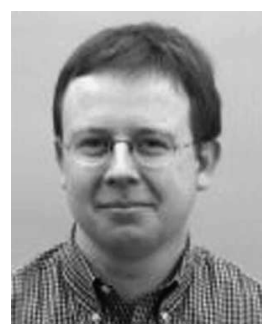

Christof Olivier was born in Izegem, Belgium, on September 24, 1976. He received the M.Sc. degree in electrical engineering and the Ph.D. degree from Ghent University, Ghent, Belgium, in July 1999 and May 2007, respectively.

From 1999 to 2005, he was working on electromagnetic field measurements around base stations for mobile communications related to the health effects of the exposure to electromagnetic radiation. His research interests are in electromagnetic field measurements, antennas, propagation, and various telecommunication technologies. Between February 2005 and August 2007, he was with Excentis, Ghent, on different broadband access technologies. Starting in September 2007, he will be with Mobistar, Brussels, as Senior Solution Engineer of wireless network design.

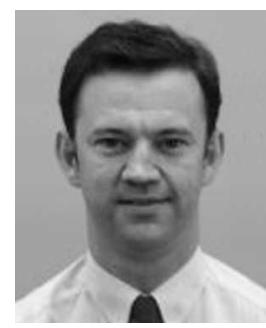

Luc Martens (M'92) was born in Ghent, Belgium, on May 14, 1963. He received the M.Sc. degree in electrical engineering and the Ph.D. degree from Ghent University in July 1986 and December 1990, respectively.

From September 1986 to December 1990, he was a Research Assistant with the Department of Information Technology (INTEC), Ghent University. During this period, his scientific work focused on the physical aspects of hyperthermic cancer therapy. His work dealt with electromagnetic and thermal modeling and with the development of measurement systems for that application. Since 1991, he has been managing the Wireless and Cable Research Group at INTEC. Since 2004, this group has been part of the interdisciplinary Institute of BroadBand Technology and focuses its research on experimental characterization of the physical layer of telecommunication systems and applications for interactive digital TV and new media for wired as well as for wireless devices. Since April 1993, he has been a Professor with Ghent University. He has authored or coauthored several publications in international conferences and journals. In 1998, he wrote a book on high-frequency characterization of electronic packaging. 